Deutsche Migräne- und Kopfschmerzgesellschaft

\title{
Kopfschmerz News
}

Wo liegt der Ursprung der Aurasymptome bei der Migräne mit Hirnstammaura (früher: Basilarismigräne)?

** Demarquay G, Ducros A, Montavont A, Mauguiere F. Migraine with brainstem aura: Why not a cortical origin? Cephalalgia 2017; doi: 10.1177/0333102417738251.

\section{Zusammenfassung}

Dieses Paper wird als Review in der Zeitschrift Cephalalgia bezeichnet, es handelt sich aber mehr um einen Diskussionsbeitrag zu der Frage, wie die Hirnstammaura entsteht. Die Autorinnen aus Frankreich versuchen aufgrund einer Literaturrecherche, die Symptome einer Hirnstammaura auf den zerebralen Kortex zurückzuführen. Sie gehen dabei so vor, dass sie jedes einzelne Symptom der Hirnstammaura, das in der IHS-Klassifikation aufgelistet ist, neuroanatomisch und neurophysiologisch diskutieren. Dies betrifft Schwindel, Störungen des Hörvermögens, Doppelbilder, Dysarthrie, Ataxie und Bewusstseinsstörungen. Sie kommen dabei zu dem Schluss, dass sämtliche Symptome einzeln auch einen kortikalen Ursprung haben können, wobei insbesondere andere paroxysmale Phänomene wie epileptische Anfälle als Erklärung herangezogen werden. Schließlich folgern die Autorinnen, dass auch die Symptome einer Hirnstammaura mit einer kortikalen Spreading Depression erklärt werden können und schlagen vor, diesen Subtyp der Migräne als normale Migräne mit Aura zu klassifizieren.

\section{Kommentar}

Das Konzept der Migräne mit Hirnstammaura (früher auch Migräne vom Basilaristyp genannt) ist seit seiner Entstehung umstritten, da eine Spreading Depression, wie sie im Tiermodell bekannt ist, im Hirnstamm kaum vorstellbar ist. Allerdings ist bis heute eine echte kortikale Spreading Depression auch beim Menschen nicht als Ursache der Migräneaura nachgewiesen.
Insofern reiht sich dieses Review ein in viele Paper, die unterschiedliche Erklärungen für eine Hirnstammaura heranziehen. Auszugehen ist erst einmal von dem Phänomen, dass es Migränepatienten gibt, die in der Tat die Symptome, die in der IHS-Klassifikation als Hirnstammsymptome aufgelistet werden, als Aura aufweisen. Der Versuch der Autorinnen ist nun leider sehr bemüht, dies auf einen kortikalen Ursprung zurückzuführen. Zwar ist in jedem Einzelfall korrekt, dass es in der wissenschaftlichen Literatur auch Beschreibungen gibt, die die Hirnstammsymptome auf einen kortikalen Ursprung zurückführen, es handelt sich dabei aber immer um Einzelfallberichte von seltenen Ereignissen, die überwiegend als epileptisch erklärt werden. Dies sei am Beispiel der Hörminderung erläutert: Eine kortikale Hörminderung ist extrem selten und nicht einmal bei größeren Hirnsubstanzschädigungen beschrieben. Es gibt sie in Einzelfällen von epileptischen Anfällen im Heschl-Gyrus, wobei es sich hier eher um eine supprimierte Hörverarbeitung als um eine echte Hypakusis handelt. Eine vorübergehende kortikale Hypakusis ist also eine Rarität. Weiterhin erklären die Autorinnen nicht, warum diese ohnehin so seltenen einzelnen kortikalen Ursprünge von klinischen Hirnstammsymptomen bei Migränepatienten dann gleichzeitig auftreten. So fordern die IHSKriterien ja das Auftreten von mindestens zwei Hirnstammsymptomen, oft sind es sogar mehr, was kortikal alleine schon statistisch extrem selten vorkommen dürfte.

Zusammenfassend ist es immer wieder hilfreich, sich über die Hirnstammaura Gedanken zu machen, da das Konzept nicht bei allen Betroffenen wirklich überzeugt. Es scheint mir aber zu einfach, die Symptome, die klinisch auf den Hirnstamm zurückzuführen sind, einfach als kortikal umzudeuten.

Stefan Evers, Coppenbrügge

\section{Die Versorgung von Patienten mit Migräne ist in Europa unzurei- chend}

Ergebnisse einer europäischen Befragung

*** Katsarava Z, Mania M, Lampl C, Herberhold J, Steiner TJ. Poor medical care for people with migraine in Europe - evidence from the Eurolight study. The Journal of Headache and Pain 2018; 19: 10.

\section{Zusammenfassung}

Vor dem Hintergrund, dass Migräne eine zwar häufige, aber häufig vernachlässigte Erkrankung ist, wurde in 10 europäischen Ländern eine Befragung durchgeführt um Unterschiede in der Versorgung von Migränepatienten zu erfassen. In Deutschland, Litauen, Italien, Luxemburg, den Niederlanden und Spanien wurden populationsbezogene Daten erhoben. In Österreich, Spanien und England wurden die Daten von konsekutiven Patienten, die einen Allgemeinarzt konsultierten, erhoben. Zusätzliche Daten kamen über Selbsthilfeorganisationen aus Spanien, Irland und den Niederlanden. Erfasst wurden die Prävalenz und Häufigkeit der Migräne, sowie die Inanspruchnahme von medizinischen Einrichtungen und die medikamentöse Therapie.

Es wurden knapp 10000 Datensätze ausgewertet. 37,5\% der Stichproben hatten eine (wahrscheinliche) Migräne, etwa ein Drittel gab mehr als 5 Attacken pro Monat an. In den populationsbezogenen Stichproben lag die Zahl derer, die wegen Migräne einen Hausarzt bzw. Spezialisten konsultiert hatten, zwischen 9,5 und $18 \%$ bzw. 3,1 und $15 \%$. Triptane wurden am häufigsten in Spanien eingesetzt (22,4\%), sonst lag die Zahl der Triptanverordnungen bei $3,4-22,4 \%$. Eine medikamentöse Prophylaxe wurde von 1,6-6,4\% der Betroffenen (Ausnahme Spanien: 13,7\%) eingenommen. Insgesamt war die Versorgung der Patienten aus den Stichproben, die bei Hausärzten (Triptane 13,6-24,5\%, Prophylaxe 4,4-9,1\%) und Selbsthilfeorganisationen (Triptane 46,2-68,2\%, Prophylaxe $16-41,7 \%)$ erhoben wurden, deutlich besser. Ausschließlich mit Selbstmedikation versorgten sich je nach Land $48-84 \%$ der Betroffenen. 
In Deutschland setzten 11\% der Betroffenen Triptane ein. Obwohl 38,5\% der Betroffenen mehr als 5 Attacken pro Monat angaben, wurden nur 2,4\% mit einer Prophylaxe behandelt. Nur knapp 20\% der Betroffenen aus der deutschen Stichprobe hatte einen Arzt konsultiert.

\section{Kommentar}

Es ist ein äußerst wichtiges Anliegen, sich ein Bild über die Qualität der ärztlichen Versorgung von Migränepatienten zu machen, da man nur so daran arbeiten kann Lücken zu schließen. Die vorgelegten Daten sind Besorgnis erregend, da sie auch in hoch entwickelten Industrieländern ganz erhebliche Defizite aufzeigen. Allerdings muss man sich vor Augen halten, dass die Datengrundlage insgesamt nicht nur heterogen, sondern ebenfalls lückenhaft ist. So lag der Anteil der Responder zwischen 11,3\% (Deutschland) und 90,7\% (Selbsthilfeorganisation Spanien). Aus der klinischen Erfahrung kann man dennoch ableiten, dass Betroffene zu selten ärztliche Hilfe aufsuchen. Die Qualität der ärztlichen Versorgung hängt häufig offenbar mit davon ab, wie aufgeklärt der Patient über die Versorgungsmöglichkeiten ist. In allen Stichproben waren die Patienten, die sich ausschließlich mit Selbstmedikation behalfen am schlechtesten versorgt. In allen europäischen Ländern haben Patienten $\mathrm{Zu}$ gang zu ärztlicher Versorgung. Dennoch ist Migräne in allen Ländern unzureichend versorgt, denn auch beim Hausarzt und beim Spezialisten erhalten Patienten nicht die bestmögliche Therapie. Die individuelle Beeinträchtigung, die mittelbaren und unmittelbaren Auswirkungen auf das Umfeld der Betroffenen und die daraus resultierenden indirekten Kosten können nur Anlass dazu sein, die Versorgung zu verbessern. Hierzu sind politische und berufspolitische Aktivitäten ebenso erforderlich, wie Aktivitäten der Fachgesellschaft und jedes Einzelnen.

Stefanie Förderreuther, München

\section{Beeinträchtigung durch Migräne}

Migräne „belegt“ Platz 1. bei Menschen unter 50 Jahre.

**** Global, regional, and national burden of neurological disorders during 1990-2015: a systematic analysis for the Global Burden of Disease Study 2015. GBD 2015 Neurological Disorders Collaborator Group. Lancet Neurol 2017; 16: 877-897.

\section{Zusammenfassung}

The „Global Burden of Disease” publizierte in Lancet 2017 eine Analyse zur Beeinträchtigung durch verschiedene neurologische Erkrankungen. Das Ranking beruht auf mehreren Indikatoren: Häufigkeit der Erkrankung, Mortalität, Disease Adjusted Life Years (DALY), Years of Life Lost (YLLs) und Years Lived with Disability (YLDs). Der YLD Index berücksichtigt sowohl die Schwere als auch die Häufigkeit der Erkrankung.

Die neurologischen Erkrankungen verantworten insgesamt 8,6\% aller Erkrankungen in der Welt. An neurologischen Erkrankungen kommen Spannungskopfschmerz, Migräne und Medikamentenübergebrauchskopfschmerz am häufigsten vor, gefolgt von Alzheimer- und anderen Demenzen. Schaut man sich das YLD Ranking an, so ist der Rückenschmerz auf Platz 1 und Migräne Platz 2. In der Population von Menschen unter dem 50. Lebensjahr ist Migräne die meist beeinträchtigende Erkrankung überhaupt.

\section{Kommentar}

1990 entstand mittels Finanzierung durch die Bill und Melinda Gates Stiftung in Seattle ein Institut für Gesundheitsforschung. Ziel der Forschung besteht in der Beschreibung und Analyse epidemiologischer und gesundheitspolitischer Daten weltweit. Alle 5 Jahre erscheint die so genannte Global Burden of Disease Studie, die alle in der Literatur zur Verfügung stehenden Studien zur Prävalenz, Inzidenz und Beeinträchtigung durch verschiedene Erkrankungen zusammenführt und aufgrund ihrer Bedeutung regelmäßig im Lancet erscheint. Mittlerweile hat das Institut eine Stellung, die mit der Weltgesundheitsorganisation vergleichbar ist.
Um verschiedene Erkrankungen miteinander vergleichen zu können werden die DALYs (disease adjusted life years) und YLDs (years lived with disability) berechnet. Mit kleineren Unterschieden geht es hierbei um mathematische Produkte aus der Schwere der Erkrankung und der Frequenz, mit welcher diese vorkommt. Hierbei sollte man einen doch möglichen Bias berücksichtigen: die GBD-Studie kann nur so gut sein, wie gut und wie oft zur bestimmten Erkrankung publiziert wurde. Sprich, liegen zu einer Erkrankung gar keine oder nur wenige Untersuchungen vor, wird diese Erkrankung im GBD-Ranking schlecht abschneiden.

Es ist ein Verdienst von der "Global Campaign Against Headache" unter der Leitung von Prof. Timothy Steiner (Imperial College London und Universität in Trondheim), dass aufgrund von vielen $\mathrm{Pu}$ blikationen zum Thema Kopfschmerz weltweit, Migräne und andere Kopfschmerzen in den letzten Jahren im GBDRanking im höher eingestuft werden. Die oben genannte Publikation berücksichtigte zum ersten Mal den Medikamentenübergebrauchskopfschmerz, was dem Kopfschmerz noch mehr Gewicht verlieh.

Desto erstaunlicher ist aber, dass eine Erkrankung, die so oft vorkommt und so viel Leiden mit sich bringt, so wenig Aufmerksamkeit seitens der Ärzte und der Gesundheitspolitik erfährt. Die Versorgung von Patienten mit Migräne ist nicht nur in Entwicklungsländern, sondern auch in wohlhabenden Europäischen Ländern schlecht $(1,2)$. Es liegt an uns selbst was zu ändern.

Zaza Katsarava, Unna

\section{Literatur}

1. Steiner TJ, Stovner LJ, Vos T, Jensen R, Katsarava Z. Migraine is first cause of disability in under 50s: will health politicians now take notice? J Headache Pain 2018; 19: 17.

2. Katsarava Z, Mania M, Lampl C, Herberhold J, Steiner TJ. Poor medical care for people with migraine in Europe - evidence from the Eurolight study. J Headache Pain 2018; 19: 10. 
Stresserleben kann Migräneanfälle vorhersagen

Bestimmte Stresssituationen können bei einer Untergruppe von Patienten Migräne auslösen. Während dies statistisch gut abgesichert werden kann, ist die Zwangsläufigkeit jedoch ungewiss.

*** Houle TT, Turner DP, Golding AN, Porter JAH, Martin VT, Penzien DB, Tegeler $\mathrm{CH}$. Forecasting individual headache attacks using perceived stress: development of a multivariable prediction model for persons with episodic migraine. Headache 2017; 57: 1041-1050.

\section{Zusammenfassung}

Es lohnt sich, nach möglichen Auslösern eines Migräneanfalls zu suchen. Neben einer effektiveren Akutbehandlung kann damit auch eine proaktive Anfallskupierung erreicht werden. Neben vielen individuellen Auslösern spielt Stress eine herausragende Rolle. Die Autoren hypothetisieren, dass Häufigkeit und Intensität aufreibender Tagesereignisse das Auftreten von Kopfschmerzen vorhersagen können.

Bei der vorgelegten Studie handelt es sich um einen prospektiven LongitudinalAnsatz auf der Basis täglicher Einschätzungen von Belastung und Kopfschmerz. Neben Angaben zum Kopfschmerz wurde das „Daily Stress Inventory" vorgelegt. Die Angaben wurden individuell über Zeitreihenanalysen ausgewertet. Erfasst wurden 95 Patienten mit Daten von insgesamt 4626 Tagen und Anfällen an 1613 Tagen, wobei an Tagen vor einem Migräneanfall vermehrt über Stressbelastungen berichtet wurde. Um eine möglichst große Passung zwischen Vorhersagemodell und tatsächlichem Auftreten von Migräne zu erreichen wurden Modelle mit unterschiedlicher Komplexität gerechnet. Die beste Passung erreichte ein Modell mit den Einflussgrößen „Häufigkeit der Belastung" und „wahrgenommene Stressintensität“. Dies konnte in einer internen Reliabilitätsanalyse bestätigt werden. Diskutiert werden die positiven Effekte einer präemptiven Strategie.

\section{Kommentar}

In der aktuellen Studie ist der Zusammenhang zwischen Stress und Migräneattacke evident, jedoch nicht zwangsläufig. So bleibt weiterhin die Ungewissheit, inwieweit Moderatorvariablen zwischen Stress und Migräne eine Rolle spielen. Das vorgestellte Modell berücksichtigt auch nicht den oft beobachteten Zusammenhang zwischen Stressabfall und Migränebeginn. Es bietet aber eine mathematisch über Zeitreihenanalysen abgesicherte Verbindung zwischen diesen beiden Faktoren. Mit größeren Studien wäre es durchaus möglich, Untergruppen darzustellen, die bei der Migräneauslösung unterschiedliche Faktoren berücksichtigen können.

So ist es das Verdienst der Arbeitsgruppe, eine weitere präemptive Therapiestrategie als neuen Weg angesprochen zu haben.

Peter Kropp, Rostock

\section{Trigeminusneuralgie bei Patienten mit Multipler Sklerose}

Neben der bekannten Assoziation mit Hirnstammläsionen beschreibt die vorliegende Studie eine Assoziation mit Läsionen der vorderen Inselrinde.

${ }^{* * *}$ Fröhlich K, Winder K, Linker RA, Engelhorn T, Dörfler A, Lee DH, Hilz M), Schwab S, Seifert F. Supratentorial lesions contribute to trigeminal neuralgia in multiple sclerosis. Cephalalgia 2017; doi: $10.1177 / 0333102417737788$.

\section{Zusammenfassung}

Die Studie ist der Frage nachgegangen, ob bei einer MS-assoziierten Trigeminusneuralgie (TGN) neben den bekannten Läsionen im Bereich der Eintrittszone des N. trigeminus im Hirnstamm auch supratentorielle Läsionen eine Rolle spielen könnten. Aus 12210 Patienten mit MS, die zwischen 2002 und 2015 das Universitätsklinikum Erlangen besucht haben, wurden retrospektiv 41 mit TGN identifiziert. 2 Patienten mit neurovaskulärem Kontakt am N. trigeminus wurden ausgeschlossen. Kontrollen waren 41 für Alter, Geschlecht und EDSS gematchte Patienten mit MS ohne TGN. T2-Aufnahmen mit manuell umfahrenen MS-Läsionen wurden auf ein gemeinsames Template normalisiert. Sowohl in einer einfachen Subtraktionsanalyse als auch in einer nicht parametrischen statistischen Auswertung fanden sich bei Patienten mit TGN häufiger Läsionen im Hirnstamm (ipsilateral im Verlauf des N. trigeminus) sowie in der kontralateralen vorderen Inselrinde. 46\% der Patienten mit TGN hatten eine Läsion in Hirnstamm und Insula, $12 \%$ eine Läsion nur im Hirnstamm, $32 \%$ eine Läsion nur in der Insula und 10\% weder noch. Für Patienten ohne TGN waren die entsprechenden Prozentsätze 10\%, $5 \%, 22 \%$ und $63 \%$. Die vordere Inselregion hat aktivierende Verbindungen zu Zentren der absteigenden Schmerzhemmung, z. B. zum periaqäduktalen Grau. Als Mechanismus schlagen die Autoren daher vor, dass Läsionen in der vorderen Insula über eine reduzierte absteigende Schmerzhemmung $\mathrm{zu}$ einer erhöhten Empfindlichkeit für Schmerzreize führen und so eine TGN begünstigen. Limitationen sind, dass aufgrund eingeschränkter Bildgebung die Detektion von neurovaskulären Kontakten nur eingeschränkt möglich war. Außerdem fand sich in der Kontrollgruppe gegenüber der TGN-Gruppe eine signifikant höhere Gesamt-Läsionslast.

\section{Kommentar}

Patienten mit MS haben ein erhöhtes Risiko für eine TGN, und diese tritt im Mittel 10 Jahre früher auf als in Fällen ohne MS. Mehrere Studien haben eine Assoziation mit Hirnstammläsionen im Verlauf des ipsilateralen $\mathrm{N}$. trigeminus gezeigt. Es gibt Hinweise darauf, dass oft sowohl eine Hirnstammläsion als auch ein Gefäß-Nervenkontakt wie bei der klassischen TGN vorliegt, so dass ein "dual hit" postuliert wurde (2). Trotzdem findet sich manchmal weder eine Hirnstammläsion noch ein Gefäß-Nervenkontakt.

Die vorliegende Studie zeigt nun eine Assoziation mit Läsionen in der kontralateralen vorderen Insula, in 32\% der Fälle ohne gleichzeitige Hirnstammläsion. Man kann sich schlecht vorstellen, dass eine Läsion in der Insula alleine zu Attacken einer TGN führt (was die Autoren auch nicht behaupten). Möglicherweise hatten doch einige dieser Patienten einen nicht detektierten Gefäß-Nervenkontakt, der zusammen mit einer Reduktion der endogenen Schmerzhemmung durch die Läsion der 
Insula dann zu Symptomen geführt haben könnte. Interessant ist auch, dass die Assoziation nicht den hinteren Teil der Insula betrifft, der mit zentralem Schmerz nach Schlaganfall assoziiert ist (1).

Aufgrund der Heterogenität der Bildgebung (erhoben über 13 Jahre, verschiedene Scanner) und der nicht sicheren Detektion eines Gefäß-Nervenkontakts müssen diese Ergebnisse sicher unabhängig bestätigt werden. Auffällig ist auch, dass insgesamt eine TGN nur bei $0,3 \%$ der Patienten mit MS gefunden wurde. Dies spricht für eine nicht systematische Erfassung dieser Beschwerden, entsprechend liegt auch keine gute klinische Charakterisierung der TGN (Erkrankungsdauer, Anzahl der Attacken etc.) vor, die man sich für zukünftige Studien wünschen würde. Insgesamt spricht aber die Reproduktion der in früheren Stu- dien gefundenen Hirnstammläsionen für die Validität der Studie, und die Assoziation in der Insula stellt einen interessanten Ansatzpunkt für das bessere Verständnis der Häufung von TGN bei Patienten mit MS dar.

Ruth Ruscheweyh, München

\section{Literatur}

1. Garcia-Larrea L, Perchet C, Creac'h C, Convers P, Peyron R, Laurent B, Mauguiere F, Magnin M. Operculo-insular pain (parasylvian pain): a distinct central pain syndrome. Brain 2010; 133: 2528-2539.

2. Truini A, Prosperini L, Calistri V, Fiorelli M, Pozzilli C, Millefiorini E, Frontoni M, Cortese A, Caramia F, Cruccu G. A dual concurrent mechanism explains trigeminal neuralgia in patients with multiple sclerosis. Neurology 2016; 86: 2094-2099.

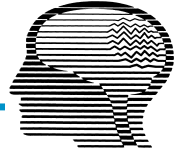

$* * * * *$ Exzellente Arbeit, die bahnbrechende Neuerungen beinhaltet oder eine ausgezeichnete Übersicht bietet **** Gute experimentelle oder klinische Studie

*** Gute Studie mit allerdings etwas geringerem Innovationscharakter ** Studie von geringerem klinischen oder experimentellen Interesse und leichteren methodischen Mängeln

* Studie oder Übersicht mit deutlichen methodischen oder inhaltlichen Mängeln

Die Kopfschmerz-News werden betreut von: Priv.-Doz. Dr. Ruth Ruscheweyh Klinik und Poliklinik für Neurologie Klinikum der Universität München Marchioninistr. 15, 81377 München Tel. 089/440073907 ruth.ruscheweyh@med.uni-muenchen.de 